\title{
Body mass index correlates with multiple sclerosis disease and symptom severity in women, but not in men
}

\author{
Richter B ${ }^{1}$, Cutter $\mathrm{G}^{3}$, Pandey $\mathrm{K}^{1}$, Mohn J ${ }^{1}$, Bacon TE ${ }^{2}$ and Kister $\mathrm{I}^{1,2 *}$ \\ ${ }^{1}$ Barnabas Health , NJ, USA \\ ${ }^{2}$ NYU Langone Medical Center, NY, USA \\ ${ }^{3} \mathrm{UAB}$ School of Public Health, AL, USA
}

\begin{abstract}
Objective: To assess correlations between Body Mass Index (BMI) and Patient-derived Multiple Sclerosis Severity Score (P-MSSS), and BMI and SymptoMScreen scores in men and women with Multiple Sclerosis (MS).

Background: Higher BMI during adolescence and young adulthood is a risk factor for MS, but data is conflicting concerning the relationship between BMI and disease severity in MS.

Methods: BMI, Patient Determined Disease Steps (PDDS), and SymptoMScreen were recorded in consecutive clinic MS patients at the NYU MS Care Center (New York, NY) and Barnabas MS Care Center (Livingston, NJ) who were relapse-free for $\geq 3$ months. P-MSSS was calculated based on PDDS and disease duration using a published reference table. Pearson correlation coefficients between P-MSSS and BMI, as well as between SymptoMScreen scores and BMI, were calculated independently for men and women. Separate logistic regression analyses were performed to evaluate the effect of age, sex, site of care and BMI on P-MSSS and symptom severity in 11 distinct domains.

Results: 1,024 patients (74.9\% women) with clinician-confirmed MS were included in the study. Men and women were similar with respect to age, BMI and disease severity scores, but men had slightly shorter disease duration than women. BMI showed a positive correlation with disease severity (P-MSSS) among women $(\mathrm{r}=0.106, \mathrm{p}=0.0043)$, but not among men $(\mathrm{r}=-0.037, \mathrm{p}=0.585)$. In a multiple regression model accounting for BMI, age, site of care and sex, BMI and age were the only significant predictors of disease severity $(\mathrm{p}<0.01)$. BMI and age were also the only significant predictors $(\mathrm{p}<0.01)$ of symptom severity in the domains of mobility, bodily pain, sensation, vision, depression, and anxiety.
\end{abstract}

Conclusions: In this cross-sectional study, BMI showed a modest correlation with MS severity and symptom burden in women. In men, only anxiety score correlated with BMI. Further study is required to determine why BMI has a sexually dimorphic effect on disease and symptom severity.

\section{Introduction}

Obesity is associated with increased levels of pro-inflammatory cytokines such as leptin and interleukin 6 [1-5], and is a risk factor for a number of autoimmune diseases [6]. Epidemiological studies have established a link between adolescent and early adulthood obesity and MS [7-10]. Recent work utilizing the technique of Mendelian randomization provide indirect evidence that obesity is a risk factor for MS $[11,12]$. The question of what effect obesity has on MS course and symptom severity has received less attention. One small-sized study demonstrated a modest correlation between obesity and disability (EDSS) [13], while a larger study showed that obesity correlated with worse disability in women, but not in men [14]. In adult MS patients, baseline body mass index (BMI) values did not predict changes in either clinician-recorded [15] or patient-recorded [16] disability over the short-term, and, in children with MS, BMI was also not associated with disease activity [17]. It is not known whether BMI has any effect on symptom burden in MS. Our work explores the relationship between BMI and patient-reported outcome measures of both disease severity (Patient-derived Multiple Sclerosis Severity Score, or P-MSSS) [18] and symptom severity (SymptoMScreen) [19] in a large sample of men and women outpatients at two MS centers.

\section{Methods}

Institutional Review Board approvals for the study were obtained from the respective institutions.

We retrospectively reviewed patient charts from consecutive MS patients seen for routine care at Barnabas MS Care Center (Livingston, NJ) and NYU MS Care Center (New York, NY). Inclusion criteria required that patients have a definite diagnosis of MS (confirmed by the treating neurologist), be relapse-free for at least three months prior to the visit recorded, and have body weight and height recorded at the time of visit. Patients self-assessed their disability with Patient Determined Disease Steps (PDDS) [20]. Patient-derived Multiple Sclerosis Severity Score (P-MSSS), a rank score of PDDS among reference MS patient population with similar disease duration, was determined using the published reference table [18]. Patients assessed their symptom

Correspondence to: Ilya Kister MD, NYU MS Care Center 240, East $38^{\text {th }}$ St New York, NY 10016, USA, E-mail: ilya.kister@gmail.com

Key words: multiple sclerosis; body masss index, sexual dimorphism, crosssectional study

Received: February 03, 2017; Accepted: February 28, 2017; Published: March 02,2017 
severity with symptoMScreen, a validated battery of Likert scales for 11 distinct domains commonly affected by MS: mobility, dexterity, spasticity, bodily pain, sensation, bladder/bowel function, fatigue, vision, cognition, depression, and anxiety (Appendix 1) [19].

Descriptive statistics and univariate analyses (Pearson correlation coefficients) were performed to assess for correlations between P-MSSS and BMI for males and females separately in view of prior observation of sex differences in the directionality of BMI associations [14]. Multiple linear regression analyses were carried out to investigate the association between BMI and disease severity (P-MSSS), and BMI and symptom severity, with age, sex, and site of care as covariates.

\section{Results}

The study sample comprised of 1,024 patients with MS, of whom $247(24.1 \%)$ were men and 777 (75.9\%) were women. Demographic and disease-related characteristics for men and women are shown in Table 1. BMI, age and disease severity (P-MSSS) were similar in the two sexes, but men had a shorter disease duration than women $(\mathrm{p}=0.042)$.

In univariate analyses, BMI positively correlated with disease severity (P-MSSS) among women $(\mathrm{r}=0.106 \mathrm{p}=0.0043)$, but not among men $(r=-0.037 p=0.585)$. In a multiple regression model calculated to predict disease severity (P-MSSS) based on BMI, age, site of care and sex, a significant regression equation was found $(\mathrm{R} 2=0.038, \mathrm{~F}$ $(4,945)=9.21)$, explaining less than $4 \%$ of the variability. BMI was a significant predictor of P-MSSS $(\mathrm{p}<0.01)$ in this model, and accounted for $2 \%$ of the variance.

Pearson correlations were assessed between BMI and symptom severity for each of the 11 domains for men and women separately. In women, significant positive correlations $(\mathrm{p}<0.05)$ were found for all domains; highest correlations coefficients were observed for depression, cognition, fatigue, sensory symptoms, anxiety and bodily pain. In men, only the anxiety domain score positively correlated with BMI (Table 2). In individual linear regression models performed for each of the 11 domains, BMI was a significant predictor of symptom severity for mobility, bodily pain, sensation, vision, depression and anxiety (Table 3). The amount of variation explained by BMI, while significant, was small. In models that tested for interactions between sex and BMI, the interaction term was $<0.075$ for mobility, hand function and cognition suggesting male/female differences in the relationship between BMI and symptom severity.

\section{Conclusions}

Our work confirms the recent observation that body mass index has a differential effect on MS severity in women and men [14]. Similar to Bove et al. [14], we found a modest correlation between obesity and disease severity (P-MSSS) in women. Bove et al. reported a significant

Table 1. Demographic characteristics of the 1024 participants by sex.

\begin{tabular}{|l|c|c|}
\hline \multicolumn{1}{|c|}{ Characteristic } & Male & Female \\
\hline Number (\%) & $247(24.1 \%)$ & $777(75.9 \%)$ \\
\hline BMI & $27.3(5.2)$ & $27.3(6.4)$ \\
\hline Age, mean (years) & $45.8(13.7)$ & $46.1(12.6)$ \\
\hline Disease Duration (years) & $11.3(11.9)$ & $13.0(9.9)$ \\
\hline P-MSSS (mean) & $4.0(2.7)$ & $3.7(2.6)$ \\
\hline Weight Classification (\%) & & \\
\hline Underweight & 1.2 & 2.8 \\
\hline Normal Weight & 33.2 & 39.3 \\
\hline Overweight & 39.7 & 29.7 \\
\hline Obese & 25.9 & 28.2 \\
\hline
\end{tabular}

Table 2. Correlation between Symptom Severity in 11 Domains and BMI: univariate analyses.

\begin{tabular}{|c|c|c|c|c|}
\hline & \multicolumn{2}{|c|}{ Female } & \multicolumn{2}{c|}{ Male } \\
\hline Domains & $\mathbf{r}$ & $\mathbf{p}$ & $\mathbf{r}$ & $\mathbf{p}$ \\
\hline Mobility & $\mathbf{0 . 1 1 7}$ & $\mathbf{0 . 0 0 1}$ & -0.023 & 0.721 \\
\hline Dexterity & $\mathbf{0 . 0 8 8}$ & $\mathbf{0 . 0 1 5}$ & -0.057 & 0.376 \\
\hline Spasticity & $\mathbf{0 . 0 8 9}$ & $\mathbf{0 . 0 1 3}$ & 0.045 & 0.488 \\
\hline Bodily pain & $\mathbf{0 . 1 2 8}$ & $<\mathbf{0 . 0 0 1}$ & 0.035 & 0.588 \\
\hline Sensation & $\mathbf{0 . 1 2 3}$ & $<\mathbf{0 . 0 0 1}$ & 0.037 & 0.564 \\
\hline Bladder function & $\mathbf{0 . 1 0 2}$ & $\mathbf{0 . 0 0 4}$ & 0.016 & 0.803 \\
\hline Fatigue & $\mathbf{0 . 1 3 0}$ & $<\mathbf{0 . 0 0 1}$ & 0.038 & 0.557 \\
\hline Vision & $\mathbf{0 . 1 0 8}$ & $\mathbf{0 . 0 0 2}$ & 0.097 & 0.133 \\
\hline Cognition & $\mathbf{0 . 1 5 5}$ & $<\mathbf{0 . 0 0 0 1}$ & 0.002 & 0.964 \\
\hline Depression & $\mathbf{0 . 1 5 1}$ & $<\mathbf{0 . 0 0 0 1}$ & 0.071 & 0.266 \\
\hline Anxiety & $\mathbf{0 . 1 2 9}$ & $<\mathbf{0 . 0 0 1}$ & $\mathbf{0 . 1 5 4}$ & $\mathbf{0 . 0 1 8}$ \\
\hline
\end{tabular}

Legend: Pearson's correlation coefficient (r) and p-value are shown for each domain; significant correlations are in bold.

Table 3. Significant Regression Equations for SymptoMScreen Domains.

\begin{tabular}{|c|c|c|c|}
\hline Domain & $\begin{array}{c}\text { Significant predictor } \\
\text { variables }\end{array}$ & Variance & P-Value \\
\hline Mobility & Age, $B M I$ & $R 2=0.11, F\{5,955)=24.75$ & $<0.001$ \\
\hline Bodily pain & Age, $B M I$ & $R 2=0.06, F\{5,985)=5.22$ & $<0.001$ \\
\hline Sensation & Age, $B M I$ & $R 2=0.05, F\{5,988)=9.44$ & $<0.001$ \\
\hline Vision & Age, $B M I$ & $R 2=0.02, F\{5,981)=3.77$ & $<0.01$ \\
\hline Depression & Age, $B M I$ & $R 2=0.03, F\{5,1001)=5.65$ & $<0.001$ \\
\hline Anxiety & Age, $B M I$ & $R 2=0.02, F\{5,965)=4.57$ & $<0.001$ \\
\hline
\end{tabular}

negative correlation between BMI and MSSS in men; in our study a non-significant, but also negative correlation was observed.

A possible explanation for higher disease severity with higher BMI is 'reverse causality': the more disabled the patients are, the less likely they are to maintain proper diet and exercise regimens and the more likely to use steroids and other medications associated with weight gain. However, this explanation might not account for sexual dimorphism with regard to MS severity and BMI relationship seen in two large studies [14]. It seems more probable that the association between disease and symptom severity and BMI among women with MS is due to higher levels of pro-inflammatory cytokines and lipidrelated variables in women with higher BMI as compared to men with comparable BMI. For instance, leptin, which is elevated in patients with progressive MS [21], is several-fold higher in women than in men [1,2]. Biological explanations for the differential relationship between BMI and disease severity in the two sexes warrants further study.

SymptoMScreen, a validated, in-house developed instrument for rapidly assessing symptom burden in MS Patients, allowed us to evaluate the relation between BMI and symptom severity in 11 distinct domains. Among women, BMI was positively correlated with higher symptom scores in every domain. The strongest correlations between BMI and disease severity were observed in the domains of mobility, bodily pain, sensation, vision, depression and anxiety. In men, only anxiety showed positive correlation with BMI. Obesity is known to be co-morbid with depression, anxiety, and pain in the general population [22-24], so it is possible that correlations we have observed are not specific to MS. Alternatively, higher symptom burden may be due to more severe disease, as reflected in higher P-MSSS scores, in women with higher BMI.

Our study relied exclusively on patient self-report for neurologic disability. It is therefore reassuring that our findings are in line with 
an earlier large-scale study that utilized clinician-rated disability [14]. The retrospective nature of our work made it impossible to control for medication use that may have affected weight (e.g. steroids) and lifestyle factors (e.g. diet, daily exercise, smoking). Despite these limitations, our finding of slightly higher disease and symptom severity among women with MS with higher BMI suggests questions for further basic science and clinical research: what are the biological mechanisms that may explain higher disease severity in higher-BMI women, but not men? Would weight loss have a symptomatically beneficial effect on MS, especially in mental health and pain domains?

\section{Disclosures}

The authors report no disclosures relevant to this work. The study was carried out using authors' own resources.

\section{References}

1. OstlundRE Jr, Yang JW, Klein S, Gingerich R (1996) Relation between plasma leptin concentration and body fat, gender, diet, age, and metabolic covariates. J Clin Endocrinol Metab 81: 3909-3913. [Crossref]

2. Rosenbaum M, Nicolson M, Hirsch J, Heymsfield SB, Gallagher D, et al. (1996) Effects of gender, body composition, and menopause on plasma concentrations of leptin. J Clin Endocrinol Metab 81: 3424-3427. [Crossref]

3. Das UN (2001) Is obesity an inflammatory condition? Nutrition 17: 953-966.[Crossref]

4. Roytblat L, Rachinsky M, Fisher A, Greemberg L, Shapira Y, et al. (2000) Raised interleukin-6 levels in obese patients. Obes Res 8: 673-675.[Crossref]

5. Mohamed-Ali V, Goodrick S, Rawesh A, Katz DR, Miles JM, et al. (1997) Subcutaneous adipose tissue releases interleukin-6, but not tumor necrosis factor-alpha, in vivo. J Clin Endocrinol Metab 82: 4196-4200.[Crossref]

6. Harpsøe, Maria C, Andersson M, Nielsen NM, Frisch M, et al. (2014) Body mass index and risk of autoimmune diseases: a study within the Danish National Birth Cohort. Int J Epidemiol 43: 843-855.[Crossref]

7. Munger KL, Chitnis T, Ascherio A (2009) Body size and risk of MS in two cohorts of US women. Neurology 73: 1543-1550.[Crossref]

8. Hedström AK, Olsson T, andAlfredsson L (2016) Body mass index during adolescence, rather than childhood, is critical in determining MS risk. Mult Scler 22: 878-883. [Crossref]

9. Kavak KS, Teter BE, Hagemeier J, Zakalik K, Weinstock-Guttman B (2015) Higher weight in adolescence and young adulthood is associated with an earlier age at multiple sclerosis onset. Mult Scler 21: 858-865.

10. Hedström AK, Olsson T, Alfredsson L (2016) Body mass index during adolescence, rather than childhood, is critical in determining MS risk. Mult Scler 22: 878-883.[Crossref]
11. Gianfrancesco MA, Glymour MM, Walter S, Rhead B, Shao X, et al. (2017) Causal Effect of Genetic Variants Associated with Body Mass Index on Multiple Sclerosis Susceptibility. Am J Epidemiol [Crossref]

12. Mokry LE, Ross S, Timpson NJ, Sawcer S, et al. (2016) Obesity and Multiple Sclerosis: A Mendelian Randomization Study. PLoS Med 13: e1002053.[Crossref]

13. Tettey P, Simpson S Jr, Taylor B, Blizzard L, Ponsonby AL, et al. (2014) An adverse lipid profile is associated with disability and progression in disability, in people with MS. Mult Scler 20: 1737-1744.[Crossref]

14. Bove R, Musallam A, Xia Z, Baruch N, Messina S, et al. (2016) Longitudinal BMI trajectories in multiple sclerosis: Sex differences in association with disease severity. Mult Scler Relat Disord 8: 136-140.[Crossref]

15. Weinstock-Guttman B, Zivadinov R, Mahfooz N, Carl E, Drake A, et al. (2011) Serum lipid profiles are associated with disability and MRI outcomes in multiple sclerosis. $J$ Neuroinflammation 8: 127.[Crossref]

16. Pilutti L, McAuley E, Motl RW (2012) Weight status and disability in multiple sclerosis: An examination of bi-directional associations over a 24-month period. Multiple sclerosis and related disorders 1: 139-144.[Crossref]

17. Krysko K, Kalina J, Ford R, Pandey K, Kister I, et al. (2016) Obesity and Disease Activity in Pediatric-Onset Multiple Sclerosis (P1. 376). Neurology 86.16 Supplement P1-376.

18. Kister I, Chamot E, Salter AR, Cutter GR, Bacon TE, et al. (2013) Disability in multiple sclerosis: a reference for patients and clinicians. Neurology 80: 1018-1024.[Crossref]

19. Green R, Kalina J, Ford R, Pandey K, Kister I, et al. (2016) SymptoMScreen: a tool for rapid assessment of symptom severity in MS across multiple domains. Appl Neuropsychol Adult 1-7. [Crossref]

20. Hohol MJD, Orav EJ, Weiner HL (1995) Disease Steps in multiple sclerosis A simple approach to evaluate disease progression. Neurology 45: 251-255.[Crossref]

21. Zarkesh-Esfahani, SH (2011) Leptin serum levels in different subtypes of Multiple sclerosis: does leptin play a role in progress of Multiple sclerosis? The Journal of Immunology 1: 44-9.

22. Luppino FS, de Wit LM, Bouvy PF, Stijnen T, Cuijpers P, et al. (2010) Overweight, obesity, and depression: a systematic review and meta-analysis of longitudinal studies. Arch Gen Psychiatry 67: 220-229.[Crossref]

23. Gariepy G, Nitka D, Schmitz N (2010) The association between obesity and anxiety disorders in the population: a systematic review and meta-analysis. Int $J$ Obes (Lond) 34: 407-419.[Crossref]

24. Hitt HC, McMillen RC, Thornton-Neaves T, Koch K, Cosby AG (2007) Comorbidity of obesity and pain in a general population: results from the Southern Pain Prevalence Study. J Pain 8: 430-436.[Crossref]

Copyright: $(02017$ Richter B. This is an open-access article distributed under the terms of the Creative Commons Attribution License, which permits unrestricted use, distribution, and reproduction in any medium, provided the original author and source are credited. 